\title{
An Electrophoretic Slow PGM1 Variant Detected in a Yamanashi Population
}

\author{
Nori Komatsu, Akira Kido and Masakazu Oya \\ Department of Legal Medicine, Yamanashi Medical \\ University, Tamaho-cho 409-38
}

Short Report

Komatsu, N., Kido, A. and Oya, M. An Electrophoretic Slow PGM1 Variant Detected in a Yamanashi Population. Tohoku J. exp. Med., 1986, 150 (3), 359-360 — Using starch gel electrophoresis, a slow variant PGM1 6NGS2-1 (W23-1A) was detected among 749 residents of Yamanashi Prefecture. The isoelectric focusing pattern of this type was demonstrated, which would serve as a reference in further population studies.—__ enzyme polymorphism; slow PGM1 variant; starch gel electrophoresis ; isoelectric focusing

The polymorphism of the first locus phosphoglucomutase (PGM1) has been extensively investigated in various populations, and a number of variant phenotypes have been discovered by different electrophoretic techniques. In 1983, an international workshop on the PGM1 system was held in Munich, where 30 different rare variants were identified and designated according to a newly-recommended nomenclature (Dykes et al. 1985). This paper reports an electrophoretic slow PGM1 variant detected among 749 residents of Yamanashi Prefecture.

Freshly-prepared haemolysates were analyzed both by conventional electrophoresis on starch gels, pH 7.4 (Spencer et al. 1964) and isoelectric focusing on polyacrylamide gels, $\mathrm{pH}$

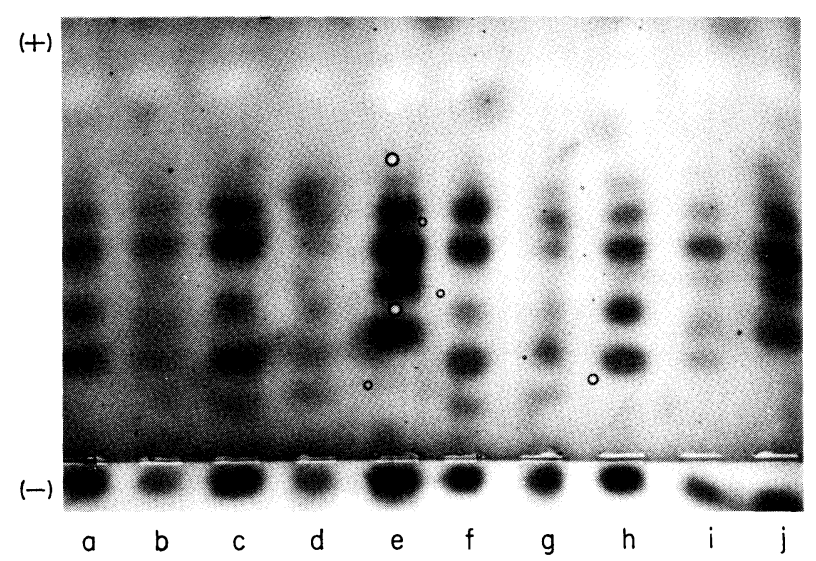

Fig. 1. PGM1 patterns obtained by starch gel electrophoresis. a and h, PGM1 1 ; b and i, PGM1 2-1; c and f, PGM1 8-1; d and g, PGM1 6NGS2-1; e and j, PGM1 2. 


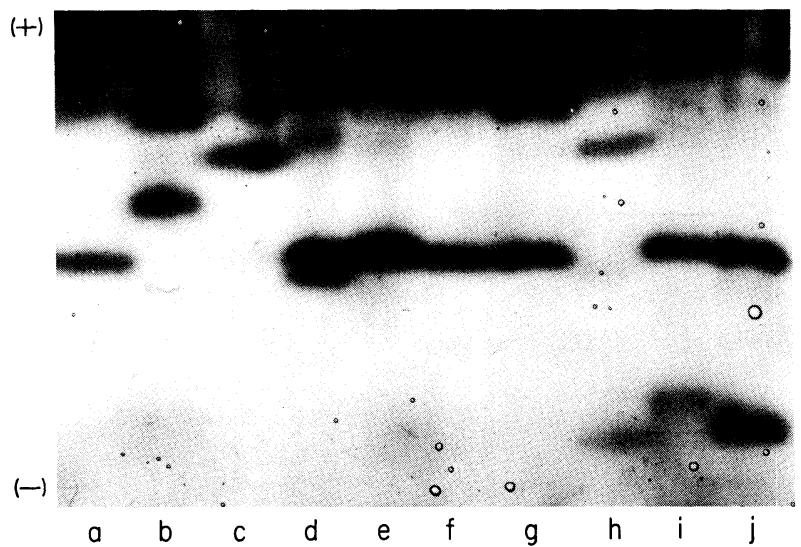

Fig. 2. PGM1 patterns obtained by polyacrylamide gel isoelectric focusing. a, e, f and g, PGM1 1A ; b, PGM1 2B ; c, PGM1 2A ; d, PGM1 1A-1B ; h, PGM1 W27-2A ; i, PGM1 W23-1A; j, PGM1 W27-1A.

\section{5-7 (Bark et al. 1976).}

The variant (positions $d$ and $g$ in Fig. 1) was determined as 6NGS2-1, based on the starch gel electrophoretic patterns reported in the previous papers (Satoh et al. $1976 ; 1984$ ). The isoelectric focusing pattern of this type (position i in Fig. 2) corresponded with that of the type W23-1A illustrated diagrammatically in the report on the International Workshop.

The allele 6NGS2 was first found by Satoh et al. (1976) in the Nagasaki population and newly designated W23 at the International Workshop. However, there has appeared no photographic representation of the isoelectric focusing pattern of the types associated with this variant allele. It may therefore be necessary to demonstrate the isoelectric focusing pattern of PGM1 W23-1A since samples must be phenotyped using a minimum of two different techniques, as suggested at the International Workshop.

\section{References}

1) Bark, J.E., Harris, M.J. \& Firth, M. (1976) Typing of the common phosphoglucomutase variants using isoelectric focusing - A new interpretation of the phosphoglucomutase system. J. forens. Sci. Soc., 16, 115-120.

2) Dykes, D.D., Kühnl, P. \& Martin, W. (1985) PGM1 system. Report on the International Workshop, October 10-11, 1983, Munich, West Germany. Amer. J. hum. Genet., 37, 1225-1231.

3) Satoh, C., Ueda, N., Horai, S. \& Omoto, K. (1976) Further studies on phosphoglucomutase-1 phenotypes in Japanese. I. Comparison of "slow" variants. Jap. J. hum. Genet., 21, 85-96.

4) Satoh, C., Takahashi, N., Kaneko, J., Kimura, Y., Fujita, M., Asakawa, J., Kageoka, T., Goriki, K. \& Hazama, R. (1984) Electrophoretic variants of blood proteins in Japanese. II. Phosphoglucomutase-1 (PGM1). Jap. J. hum. Genet., 29, 287-310.

5) Spencer, N., Hopkinson, D.A. \& Harris., H. (1964) Phosphoglucomutase polymorphism in man. Nature (Lond.), 204, 742-745. 